\title{
Ardisia crenata extract stimulates melanogenesis in B16F10 melanoma cells through inhibiting ERK1/2 and Akt activation
}

\author{
CHENG YAO ${ }^{1-3}$, CHENG LONG JIN ${ }^{1-4}$, JANG-HEE OH ${ }^{1-3}$, INN GYUNG OH ${ }^{1-3}$, \\ CHI-HYUN PARK ${ }^{1-3^{*}}$ and JIN HO CHUNG ${ }^{1-3^{*}}$
}

\author{
${ }^{1}$ Department of Dermatology, Seoul National University College of Medicine; \\ ${ }^{2}$ Institute of Human-Environment Interface Biology, Medical Research Center, Seoul National University, Seoul 110-744; \\ ${ }^{3}$ Laboratory of Cutaneous Aging Research, Biomedical Research Institute, Seoul National University Hospital, \\ Seoul 110-744, Republic of Korea; ${ }^{4}$ Department of Dermatology, Yanbian University Hospital, Yanji, Jilin 133000, P.R. China
}

Received November 21, 2013; Accepted March 17, 2014

DOI: $10.3892 / \mathrm{mmr} .2014 .2697$

\begin{abstract}
Melanin protects the skin against ultraviolet radiation by scattering incoming light and absorbing diverse free radicals. Agents that increase melanin synthesis in melanocytes may reduce the risk of photodamage and skin cancer. The present study investigated the effect of a methanol extract of Ardisia crenata (AC) on melanogenesis in B16F10 cells. Treatment of cultured B16F10 cells with AC extract (10, 20 and $40 \mu \mathrm{g} / \mathrm{ml}$ ) stimulated an increase in melanin levels in a concentration-dependent manner, without cytotoxicity. Tyrosinase is key in the regulation of melanin production, thus the effect of $\mathrm{AC}$ extract on tyrosinase activity and protein expression was analyzed. AC extract was observed to significantly increase tyrosinase activity and protein expression in B16F10 cells. Furthermore, AC extract was found to markedly increase the protein expression of microphthalmia-associated transcription factor, which is an important transcription factor involved in tyrosinase gene expression. In addition, AC extract
\end{abstract}

Correspondence to: Dr Jin Ho Chung, Department of Dermatology, Seoul National University College of Medicine, Seoul National University Hospital, 28 Yongon-dong, Chongno-gu, Seoul 110-744, Republic of Korea

E-mail: jhchung@snu.ac.kr

${ }^{*}$ Contributed equally

Abbreviations: $\alpha$-MSH, $\alpha$-melanocyte stimulating hormone; MITF, microphthalmia-associated transcription factor; AC, Ardisia crenata; MTT, 3-(4,5-dimethylthiazol-2-yl)-2,5-diphenyltetrazolium bromide; PKA, protein kinase A; CREB, cyclic adenosine monophosphate response element binding protein; L-DOPA, L-3,4-dihydroxyphenylalanine; TRP, tyrosinase-related protein; ERK, extracellular signal-regulated kinases

Key words: Ardisia crenata extract, melanin, tyrosinase, microphthalmia-associated transcription factor, extracellular signal-regulated kinases, Akt
(40 $\mu \mathrm{g} / \mathrm{ml}$ ) was observed to suppress the activation of extracellular signal-regulated kinase (ERK) and Akt, which negatively regulate melanin synthesis in B16F10 cells. In conclusion, to the best of our knowledge, the present study is the first to show that a methanol extract of AC stimulates melanogenesis by increasing tyrosinase expression via the inhibition of ERK and Akt. Thus, methanol extract of AC may be a potential treatment for hypopigmentation diseases and may be a candidate for skin-tanning cosmetic products.

\section{Introduction}

Melanogenesis is a physiological process, which is involved in the production of melanin. Melanin is synthesized within membrane-bound organelles, termed melanosomes, in melanocytes and is transferred to keratinocytes where it forms melanin caps above keratinocyte nuclei (1). Keratinocytes and melanosomes provide strong protection against ultraviolet radiation (UVR)-induced photodamage by scattering incoming light and absorbing diverse free radicals in cells (2). Furthermore, reduced or defective melanin pigmentation is associated with an increased risk of skin cancer and various other pathological conditions (3-4).

In melanocytes and melanoma cells, melanin synthesis is primarily controlled through an enzymatic cascade that is regulated by tyrosinase, tyrosinase-related protein 1 and tyrosinase-related protein 2 (5). Tyrosinase is the rate-limiting enzyme, which is critical in the regulation of melanin production. Tyrosinase catalyzes the hydroxylation of tyrosine to 3,4-dihydroxyphenylalanine (DOPA) and the oxidation of DOPA to dopaquinone (6). In addition, one of the most important transcription factors involved in the regulation of tyrosinase is microphthalmia-associated transcription factor (MITF), which has been reported to bind to the M-box within the tyrosinase promoter, thus enhancing tyrosinase gene expression (7).

Cyclic adenosine monophosphate (cAMP) is involved in various signal transduction pathways and has been reported to have a role in the regulation of melanogenesis (8). The mechanism by which cAMP regulates melanogenesis involves the activation of protein kinase A (PKA). Activated PKA 
translocates to the nucleus where it phosphorylates the cAMP responsive element-binding protein (CREB). Phosphorylated CREB subsequently binds to the CRE site on the MITF promoter and interacts with the CREB binding protein to increase the expression of MITF, resulting in melanogenesis (9-10). Various factors lead to an increase in intracellular cAMP, including $\alpha$-melanocyte stimulating hormone, forskolin and isobutyryl xanthine, and are capable of inducing melanogenesis in melanocytes and melanoma cells (11). Thus, chemicals or plant extracts, which modulate intracellular cAMP levels, are considered to be capable of regulating melanogenesis in human and mouse melanocytes (12-13).

The extracellular signal-regulated kinase (ERK) and phosphatidylinositol 3-kinase (PI3K)/Akt signaling pathways have been shown to negatively regulate melanogenesis in melanocytes and melanoma cells (14-15). Furthermore, numerous agents have been identified that upregulate melanogenesis in B16F10 cells via inhibition of the ERK and/or Akt signaling pathways. For example, lupenone and fluvastatin were reported to increase melanin synthesis by inhibiting the activation of ERK and Akt, respectively (16-17).

Stimulation of melanin synthesis has been proposed as a defense mechanism to prevent UVR-induced DNA damage in human skin and the stimulation of melanin synthesis is used to treat various diseases, which are characterized by a lack of skin pigmentation, including vitiligo (18). Due to the increasing demand to overcome the UVR-associated increase in skin cancer risk, as well as hypopigmentation diseases, including vitiligo, the development of novel, natural plant extract-derived tanning cosmetics has attracted much research attention. Extracts from certain herbs, including mangosteen (13), Erica multiflora (16), Pyrostegia venusta (19) and Daphne gnidium (20) have been shown to increase melanogenesis in B16 melanoma cells, thus these extracts may have potential for use in tanning cosmetics.

Ardisia crenata (AC) is a species of flowering plant from the Ardisia genus and the Myrsinaceae family, which is native to East Asia and commonly used as an ornamental plant. The root extracts of $\mathrm{AC}$ have been used in traditional Chinese medicine for the treatment of certain diseases, including tonsillitis, respiratory tract infections and menstrual disorders (21-22). Furthermore, various constituents of AC were reported to have significant anti-metastatic effects in tumors (23), as well as vasorelaxant effects on the aortic artery of rats (24). However, despite numerous studies in various fields, the effect of AC on melanogenesis has yet to be elucidated. The present study aimed to investigate the effect of a methanol extract of $\mathrm{AC}$ on melanin synthesis in B16F10 cells as well as the underlying molecular mechanisms involved.

\section{Materials and methods}

Materials. 3-(4,5-dimethylthiazol-2-yl)-2,5-diphenyl tetrazolium bromide (MTT), forskolin and L-DOPA, were purchased from Sigma-Aldrich (St. Louis, MO, USA). Antibodies specific to phosphorylated (P)-ERK1/2 (Thr202/Tyr204; catalog no. 9101S), total (T) ERK1/2 (catalog no. 9102), P-Akt (Ser473; catalog no. 9271) and T-Akt (catalog no. 9272) were purchased from Cell Signaling Technology Inc. (Beverly, MA, USA). Antibodies against tyrosinase and $\beta$-actin were purchased from Santa Cruz Biotechnology, Inc. (Santa Cruz, CA, USA) and anti-MITF antibodies were purchased from NeoMarkers Inc. (Fremont, CA, USA). B16F10 mouse melanoma cells were obtained from the Korean Cell Line Bank (Seoul, Korea).

Preparation of AC extract. Leaves and small branches from AC plants, which were more than three-years old and present in all areas of Jeju Island (Korea) were harvested, dried in the shade at room temperature, and stored in a dark and cold room until required. The dried plant material was extracted twice using methanol (20-times the mass of the dried material) for $72 \mathrm{~h}$ at $25^{\circ} \mathrm{C}$. The methanol AC extract was subsequently passed through $0.45-\mu \mathrm{m}$ filter paper and evaporated at $60^{\circ} \mathrm{C}$. The viscous residue was lyophilized to yield the product. Dimethyl sulfoxide (DMSO) was used to dissolve the product in order to produce the stock solution.

Cell culture. B16F10 mouse melanoma cells were cultured in phenol red-free Dulbecco's modified Eagle's medium (DMEM) supplemented with glutamine $(2 \mathrm{mmol} / \mathrm{l})$, penicillin (400 U/ml), streptomycin (50 g/l) and 10\% fetal bovine serum (FBS) at $37^{\circ} \mathrm{C}$ in a humidified atmosphere containing $5 \% \mathrm{CO}_{2}$.

Cell viability assay. Cell viability was assessed using an MTT-based assay. B16F10 cells were incubated overnight with DMEM (phenol red-free) containing 10\% FBS. Cells were subsequently treated with various concentrations of $\mathrm{AC}$ extract for $48 \mathrm{~h}$. Following treatment, $0.5 \mathrm{~g} / \mathrm{l}$ MTT dissolved in phosphate-buffered saline (PBS) was added and cells were incubated for an additional $3 \mathrm{~h}$. The supernatant was removed and DMSO was added to dissolve the formazan crystals. Absorbance was measured at $570 \mathrm{~nm}$ using a microplate reader (VersaMax; Molecular Devices, LLC., Sunnyvale, CA, USA).

Analysis of melanin content. Melanin content was measured as described previously, with slight modifications (25). B16F10 cells were incubated overnight with DMEM (phenol red-free) containing $10 \%$ FBS. Cells were treated with various concentrations of AC extract for $48 \mathrm{~h}$. Following treatment, $100 \mu \mathrm{l}$ aliquots of the media were placed in 96-well plates and the optical density (OD) was read at $405 \mathrm{~nm}$ using a microplate reader. Cells were scraped from the dishes, lysed in cell lysis buffer and protein concentration was determined using the Bradford assay (Bio-Rad, Hercules, CA, USA). Relative melanin production was calculated by normalizing the OD values with the protein concentrations (absorbance/ $\mu \mathrm{g}$ protein).

Analysis of tyrosinase activity. B16F10 cells were incubated with various concentrations of $\mathrm{AC}$ extract for $48 \mathrm{~h}$, washed with ice-cold PBS and lysed with PBS containing 1\% Triton X-100. Following centrifugation (5424R; Eppendorf, Hamburg, Germany) at $15,000 \mathrm{x} \mathrm{g}$ for $10 \mathrm{~min}$, the supernatants were collected. The quantity of each cell lysate was adjusted using lysis buffer to generate equal protein concentrations. A total of $90 \mu \mathrm{l}$ each lysate and $10 \mu \mathrm{l}$ L-DOPA $(10 \mathrm{mmol} / \mathrm{l})$ was combined in the well of a 96-well plate. Control wells contained $90 \mu \mathrm{l}$ lysis buffer and $10 \mu \mathrm{l}$ L-DOPA $(10 \mathrm{mmol} / \mathrm{l})$. Absorbance was measured at $475 \mathrm{~nm}$ using a microplate 
reader (VersaMax; Molecular Devices, LLC) subsequent to incubation at $37^{\circ} \mathrm{C}$ for $30 \mathrm{~min}$.

Western blot analysis. For the analysis of MITF and tyrosinase proteins, B16F10 cells were treated with AC extract for 24 and $48 \mathrm{~h}$, respectively. For P- or T-ERK1/2 and P- or T-Akt protein expression analysis, cells were treated with $\mathrm{AC}$ extract, and harvested at 1/3, 2, 4 and $8 \mathrm{~h}$. After the $\mathrm{B} 16 \mathrm{~F} 10$ cells were harvested, the cells were lysed in cell lysis buffer [20 mmol/1 Tris- $\mathrm{HCl}(\mathrm{pH} \mathrm{7.5),} 150 \mathrm{mmol} / \mathrm{l} \mathrm{NaCl}$, $1 \mathrm{mmol} / 1 \mathrm{EDTA}, 1 \mathrm{mmol} / 1$ ethylene glycol tetraacetic acid, $1 \%$ Triton $\mathrm{X}-100,2.5 \mathrm{mmol} / \mathrm{l}$ sodium pyrophosphate, $1 \mathrm{mmol} / \mathrm{l}$ $\beta$-glycerophosphate, $1 \mathrm{mmol} / 1 \mathrm{Na} 3 \mathrm{VO} 4,1 \mathrm{mmol} / 1$ dithiothreitol, $0.01 \mathrm{~g} / 1$ leupeptin and $1 \mathrm{mmol} / 1$ phenylmethylsulfonyl fluoride]. Equal quantities of total protein were loaded onto $8 \%$ SDS-polyacrylamide gels. Separated proteins were transferred onto polyvinylidene difluoride membranes (Roche Diagnostics GmbH, Mannheim, Germany), which were washed with 5\% dry milk in Tris-buffered saline containing $0.4 \%$ Tween 20 . The membranes were incubated with primary antibodies, followed by further incubation with horseradish peroxidase-conjugated secondary antibodies. Antibody-bound proteins were visualized using enhanced chemiluminescence (Amersham Biosciences UK Ltd., Little Chalfont, UK).

Statistical analysis. Statistical significance was determined using Student's t-test. Data are presented as the mean \pm standard deviation. $\mathrm{P}<0.05$ was considered to indicate a statistically significant difference.

\section{Results}

AC extract treatment does not induce cytotoxicity in B16F10 cells. To determine whether AC extract has a cytotoxic effect on B16F10 cells, B16F10 cells were treated with AC extract for $48 \mathrm{~h}$ at various concentrations, ranging between 10 and $40 \mu \mathrm{g} / \mathrm{ml}$. Cell viability was assessed using an MTT-based assay. AC extract was observed to have no significant effect on cell viability (Fig. 1). This finding indicates that $\mathrm{AC}$ extract is not cytotoxic to B16F10 cells at the concentrations used in the present study.

AC extract induces melanogenesis in B16F10 cells. To assess the effect of AC extract on melanogenesis, melanin levels were analyzed in B16F10 cells treated with AC extract at concentrations between 10 and $40 \mu \mathrm{g} / \mathrm{ml}$. Melanin levels were found to be significantly increased in a dose-dependent manner by $\mathrm{AC}$ extract treatment $(\mathrm{P}<0.05$; Fig. 2). Forskolin, a well-established melanogenesis inducer, served as a positive control. These findings indicate that AC extract induces melanogenesis in B16F10 cells.

AC extract induces tyrosinase activity in B16F10 cells. To investigate the possible mechanisms responsible for the $\mathrm{AC}$ extract-induced increase in melanogenesis in B16F10 cells, the effect of AC extract on tyrosinase activity was assessed. Tyrosinase is a rate-limiting enzyme in melanin synthesis. B16F10 cells were treated with AC extract at the indicated concentrations for $48 \mathrm{~h}$ and intracellular tyrosinase activity was analyzed. Forskolin served as a positive control. Fig. 3

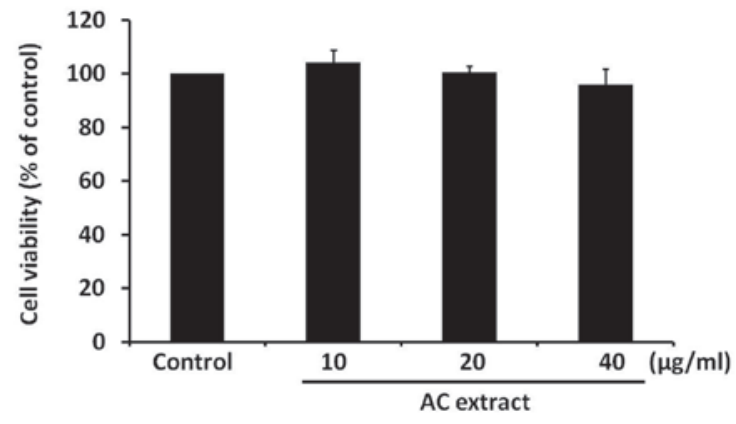

Figure 1. Effect of AC extract on B16F10 cell viability. B16F10 cells were treated with $\mathrm{AC}$ extract at the indicated concentrations. Cell viability was assessed using an MTT-based assay $48 \mathrm{~h}$ after AC extract treatment. Vehicle only served as a control. Data are presented as the mean \pm standard deviation of three independent experiments performed in triplicate. AC, Ardisia crenata.

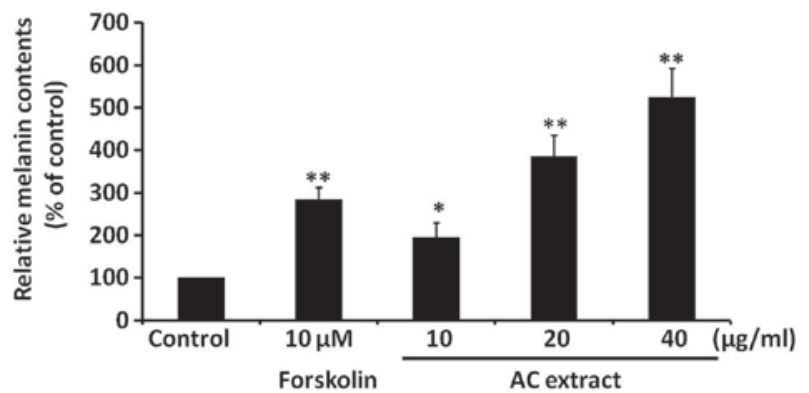

Figure 2. AC extract induces melanogenesis in B16F10 cells. B16F10 cells were treated with $\mathrm{AC}$ extract at the indicated concentrations. Relative melanin content was assessed $48 \mathrm{~h}$ after AC extract treatment. Forskolin served as a positive control and vehicle only served as a control. Data are presented as the mean \pm standard deviation of three independent experiments performed in triplicate. ${ }^{*} \mathrm{P}<0.05,{ }^{* *} \mathrm{P}<0.01$ vs. the control. AC, Ardisia crenata.

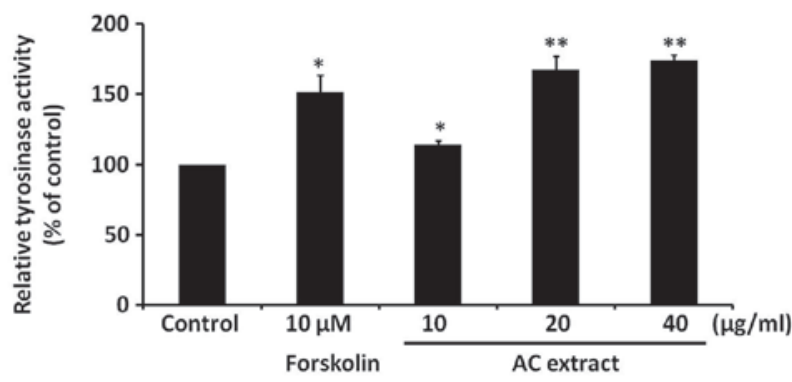

Figure 3. AC extract increases tyrosinase activity in B16F10 cells. B16F10 cells were treated with AC extract at the indicated concentrations. Intracellular tyrosinase activity was measured $48 \mathrm{~h}$ after AC extract treatment. Forskolin served as a positive control and vehicle only served as a control. Data are presented as the mean \pm standard deviation of three independent experiments performed in triplicate. ${ }^{*} \mathrm{P}<0.05,{ }^{* *} \mathrm{P}<0.01$ vs. the control. AC, Ardisia crenata.

demonstrates that AC extract significantly induced intracellular tyrosinase activity in a dose-dependent manner $(\mathrm{P}<0.05)$.

AC extract increases expression of tyrosinase and MITF in B16F10 cells. In order to clarify the mechanism underlying $\mathrm{AC}$ extract-induced tyrosinase activation, tyrosinase protein expression was assessed in B16F10 cells using western blot 
A

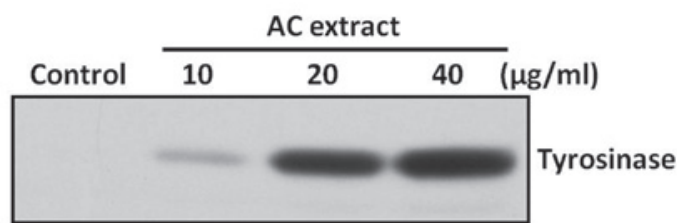

B

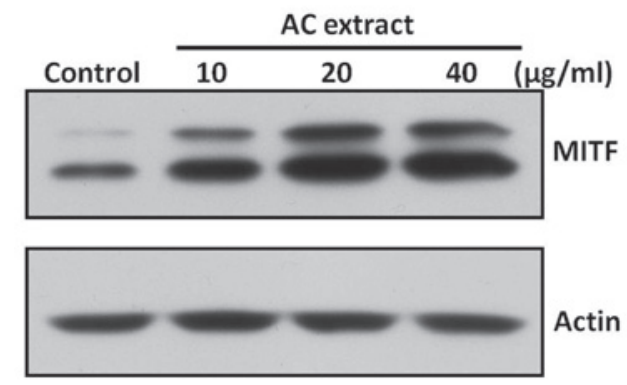

Figure 4. AC extract increases expression of tyrosinase and MITF in B16F10 cells. B16F10 cells were treated with AC extract at the indicated concentrations. (A) Tyrosinase and (B) MITF expression was analyzed 48 and $24 \mathrm{~h}$ after AC extract treatment, respectively, using western blot analysis. Equal protein loading was confirmed using anti-actin antibodies. Vehicle only served as a control. Data are representative of three independent experiments AC, Ardisia crenata; MITF, microphthalmia-associated transcription factor.

analysis. AC extract was observed to markedly increase tyrosinase protein expression (Fig. 4A). MITF is a major transcription factor for tyrosinase expression (7), therefore, the effect of AC extract on the expression of MITF was also investigated. As shown in Fig. 4B, AC extract was found to increase MITF protein expression.

$A C$ extract suppresses the activation of ERK and Akt in B16F10 cells. The ERK and the Akt signaling pathways have been shown to negatively regulate melanogenesis in melanocytes and melanoma cells (14-15). Furthermore, inhibition of ERK and PI3K/Akt has been reported to stimulate melanogenesis (26-27). Thus, the affect of AC extract on the ERK and Akt pathways in B16F10 cells was analyzed. B16F10 cells were treated with AC extract $(40 \mu \mathrm{g} / \mathrm{ml})$ for the indicated durations. Activation of the ERK or Akt signaling pathways was determined using western blot analysis with specific antibodies against phosphorylated forms of ERK and Akt. As shown in Fig. 5, ERK and Akt phosphorylation was inhibited by AC treatment at all time points.

\section{Discussion}

In the present study, the effect of AC extract on melanogenesis was investigated using a melanin content assay, intracellular tyrosinase activity assay and western blot analysis. AC extract was found to upregulate melanin synthesis in a concentration-dependent manner $(10-40 \mu \mathrm{g} / \mathrm{ml})$ without inducing cytotoxicity in B16F10 cells (Fig. 1 and 2). Tyrosinase is a key enzyme involved in melanogenesis (6), therefore, the effect of $\mathrm{AC}$ extract on tyrosinase activity and expression was also

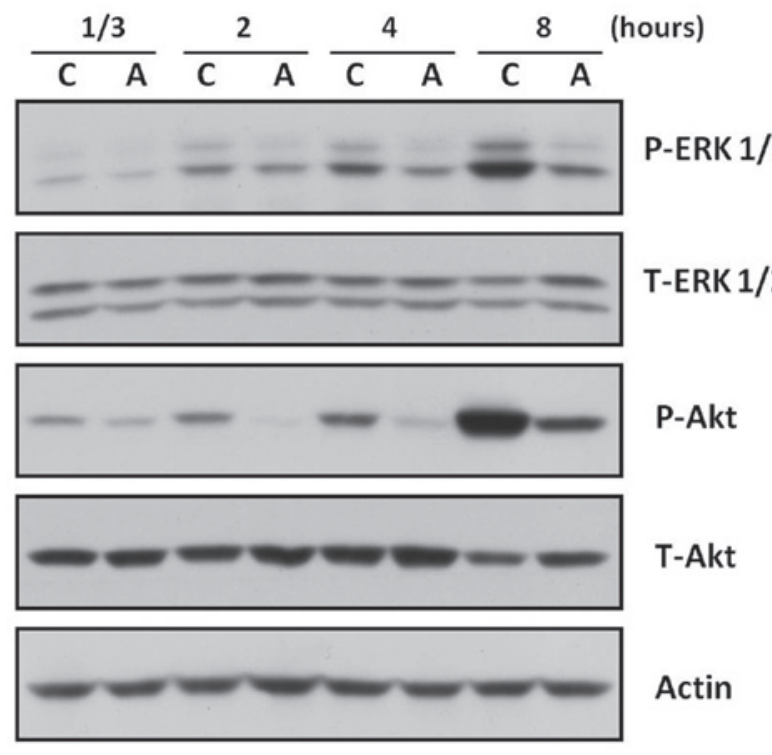

Figure 5. AC extract supresses the activation of ERK and Akt in B16F10 cells. B16F10 cells were treated with vehicle only or $40 \mu \mathrm{g} / \mathrm{ml}$ AC extract. Cells were then incubated and harvested at the indicated time points. Whole cell lysates were subjected to western blot analysis using antibodies against P- or T-ERK1/2 and P- or T-Akt. Equal protein loading was confirmed using anti-actin antibodies. Vehicle only served as a control. Data are representative of three independent experiments. AC, Ardisia crenata; ERK, extracellular signal-regulated kinase; C, control; A, AC extract; P-, phosphorylated; T-, total.

analyzed. AC extract was observed to increase tyrosinase activity and expression (Figs. 3 and 4A). Furthermore, as shown in Fig. 4B, AC extract was found to increase the expression of MITF, a transcription factor which controls pigmentation through regulating the expression of melanogenic enzymes, including tyrosinase (7).

It is well established that the ERK signaling pathway is involved in cell proliferation and differentiation (28-29). Furthermore, the ERK signaling pathway has been identified as a negative regulator of melanin synthesis and activation of the ERK signaling pathway has been reported to lead to MITF protein degradation, thus reducing melanogenesis (30). Stimulation of c-kit signaling has also been reported to induce MITF protein degradation, which is prevented by the MAPK/ERK signaling pathway inhibitor, PD98059 (30). Moreover, inhibition of the ERK signaling pathway by PD98059 has been found to induce melanogenesis in B16F10 cells (25). It is well established that the PI3K/Akt signaling pathway is important in the regulation of various cellular processes, including cell growth and apoptosis (31-32). The PI3K/Akt signaling pathway has also been shown to be involved in melanogenesis, with inhibition of the PI3K/Akt signaling pathway observed to stimulate melanogenesis in G361 melanoma and B16F10 cells (26,33). Furthermore, a previous study demonstrated that the PI3K/Akt signaling pathway inhibitor, LY294002 upregulated MITF protein expression, which increased tyrosinase expression, resulting in increased melanogenesis in B16F10 cells (33).

In order to identify the mechanisms underlying the $\mathrm{AC}$ extract-induced increase in melanogenesis in B16F10 cells, the effect of AC extract on the ERK and Akt signaling pathways was assessed. As shown in Fig. 5, AC extract inhibited ERK 
and Akt activation as early as 20 min after AC extract treatment; this inhibition was sustained for $\geq 8 \mathrm{~h}$. These findings indicate that the inhibition of ERK and/or Akt by AC extract may contribute to AC extract-induced melanogenesis through upregulating the protein expression of MITF and tyrosinase.

In conclusion, the present study has demonstrated the melanogenic effect of a methanol extract of AC in B16F10 cells and the underlying mechanisms involved. The findings indicate that $\mathrm{AC}$ extract may be useful for the treatment of hypopigmentation disorders and the development of self-tanning cosmetic products.

\section{Acknowledgements}

The authors would like to thank Bioland Co. Ltd (Cheonan, Korea) for preparation of the reagents. The present study was partially supported by grants from the National Research Foundation of Korea (the Korean government; grant no. 2011-0029819) and the Korean Health Technology R\&D Project (grant no. A121851).

\section{References}

1. Hearing VJ: Biogenesis of pigment granules: a sensitive way to regulate melanocyte function. J Dermatol Sci 37: 3-14, 2005.

2. Brenner M and Hearing VJ: The protective role of melanin against UV damage in human skin. Photochem Photobiol 84: 539-549, 2008.

3. Slominski A, Tobin DJ, Shibahara S and Wortsman J: Melanin pigmentation in mammalian skin and its hormonal regulation. Physiol Rev 84: 1155-1228, 2004.

4. Slominski A: Neuroendocrine activity of the melanocyte. Exp Dermatol 18: 760-763, 2009.

5. del Marmol V and Beermann F: Tyrosinase and related proteins in mammalian pigmentation. FEBS Lett 381: 165-168, 1996.

6. Hearing VJ and Jiménez M: Mammalian tyrosinase - the critical regulatory control point in melanocyte pigmentation. Int J Biochem 19: 1141-1147, 1987.

7. Bentley NJ, Eisen T and Goding CR: Melanocyte-specific expression of the human tyrosinase promoter: activation by the microphthalmia gene product and role of the initiator. Mol Cell Biol 14: 7996-8006, 1994.

8. Buscà R and Ballotti R: Cyclic AMP a key messenger in the regulation of skin pigmentation. Pigment Cell Res 13: 60-69, 2000.

9. Saito H, Yasumoto K, Takeda K, Takahashi K, Yamamoto H and Shibahara S: Microphthalmia-associated transcription factor in the Wnt signaling pathway. Pigment Cell Res 16: 261-265, 2003.

10. Widlund HR and Fisher DE: Microphthalamia-associated transcription factor: a critical regulator of pigment cell development and survival. Oncogene 22: 3035-3041, 2003

11. Koo JH, Kim HT, Yoon HY, et al: Effect of xanthohumol on melanogenesis in B16 melanoma cells. Exp Mol Med 40: 313-319, 2008

12. Jiang Z, Li S, Liu Y, Deng P, Huang J and He G: Sesamin induces melanogenesis by microphthalmia-associated transcription factor and tyrosinase up-regulation via cAMP signaling pathway. Acta Biochim Biophys Sin (Shanghai) 43: 763-770, 2011.

13. Hamid MA, Sarmidi MR and Park CS: Mangosteen leaf extract increases melanogenesis in B16F1 melanoma cells by stimulating tyrosinase activity in vitro and by up-regulating tyrosinase gene expression. Int J Mol Med 29: 209-217, 2012.
14. Oka M, Nagai $H$, Ando $H$, et al: Regulation of melanogenesis through phosphatidylinositol 3-kinase-Akt pathway in human G361 melanoma cells. J Invest Dermatol 115: 699-703, 2000.

15. Kim DS, Kim SY, Chung JH, Kim KH, Eun HC and Park KC: Delayed ERK activation by ceramide reduces melanin synthesis in human melanocytes. Cell Signal 14: 779-785, 2002.

16. Villareal MO, Han J, Matsuyama K, et al: Lupenone from Erica multiflora leaf extract stimulates melanogenesis in B16 murine melanoma cells through the inhibition of ERK1/2 activation. Planta Med 79: 236-243, 2013.

17. Galus R, Niderla J, Sladowski D, Sajjad E, Włodarski K and Jóźwiak J: Fluvastatin increases tyrosinase synthesis induced by alpha-melanocyte-stimulating hormone in B16F10 melanoma cells. Pharmacol Rep 62: 164-169, 2010.

18. Grimes PE: New insights and new therapies in vitiligo. JAMA 293: 730-735, 2005.

19. Moreira CG, Horinouchi CD, Souza-Filho CS, et al: Hyperpigmentant activity of leaves and flowers extracts of Pyrostegia venusta on murine B16F10 melanoma. J Ethnopharmacol 141: 1005-1011,2012.

20. Chaabane F, Pinon A, Simon A, Ghedira K and Chekir-Ghedira L: Phytochemical potential of Daphne gnidium in inhibiting growth of melanoma cells and enhancing melanogenesis of B16-F0 melanoma. Cell Biochem Funct 31: 460-467, 2013.

21. Maotian W, Xiongtai G, Xiuwen H and Shanhai H: A new triterpenoid saponin from Ardisia crenata. Planta Med 58: 205-207, 1992.

22. Jia Z, Koike K, Ohmoto T and Ni M: Triterpenoid saponins from Ardisia crenata. Phytochemistry 37: 1389-1396, 1994.

23. Wang X, Tang S, Zhai H and Duan H: Studies on anti-tumor metastatic constituents from Ardisia crenata. Zhongguo Zhong Yao Za Zhi 36: 881-885, 2011 (In Chinese).

24. Zaima K, Deguchi J, Matsuno Y, Kaneda T, Hirasawa Y and Morita H: Vasorelaxant effect of FR900359 from Ardisia crenata on rat aortic artery. J Nat Med 67: 196-201, 2013.

25. Yao C, Oh JH, Oh IG, Park CH and Chung JH: [6]-Shogaol inhibits melanogenesis in B16 mouse melanoma cells through activation of the ERK pathway. Acta Pharmacol Sin 34: 289-294, 2013

26. Buscà R, Bertolotto $\mathrm{C}$, Ortonne JP and Ballotti R: Inhibition of the phosphatidylinositol 3-kinase/p70(S6)-kinase pathway induces B16 melanoma cell differentiation. J Biol Chem 271: 31824-31830, 1996.

27. Englaro W, Bertolotto C, Buscà R, et al: Inhibition of the mitogen-activated protein kinase pathway triggers B16 melanoma cell differentiation. J Biol Chem 273: 9966-9970, 1998.

28. Cowley S, Paterson H, Kemp P and Marshall CJ: Activation of MAP kinase kinase is necessary and sufficient for PC12 differentiation and for transformation of NIH 3T3 cells. Cell 77: 841-852,1994.

29. Sale EM, Atkinson PG and Sale GJ: Requirement of MAP kinase for differentiation of fibroblasts to adipocytes, for insulin activation of p90 S6 kinase and for insulin or serum stimulation of DNA synthesis. EMBO J 14: 674-684, 1995.

30. Wu M, Hemesath TJ, Takemoto CM, et al: c-Kit triggers dual phosphorylations, which couple activation and degradation of the essential melanocyte factor Mi. Genes Dev 14: 301-312, 2000.

31. AhmadS,SinghNandGlazerRI:Role of AKT1 in 17beta-estradioland insulin-like growth factor I (IGF-I)-dependent proliferation and prevention of apoptosis in MCF-7 breast carcinoma cells. Biochem Pharmacol 58: 425-430, 1999.

32. Tang Y, Zhou H, Chen A, Pittman RN and Field J: The Akt proto-oncogene links Ras to Pak and cell survival signals. J Biol Chem 275: 9106-9109, 2000.

33. Khaled M, Larribere L, Bille K, Ortonne JP, Ballotti R and Bertolotto C: Microphthalmia associated transcription factor is a target of the phosphatidylinositol-3-kinase pathway. J Invest Dermatol 121: 831-836, 2003. 